\title{
A Plasmonic Sensor Based on D-Shaped Dual-Core Microchannel Photonic Crystal Fiber
}

Pibin Bing ( $\sim$ bing463233@163.com )

Electric Power https://orcid.org/0000-0003-0059-135X

Qing Liu

North China University of Water Resources and Electric Power

\section{Guifang Wu}

North China University of Water Resources and Electric Power

Sheng Yuan

North China University of Water Resources and Electric Power

Zhongyang Li

North China University of Water Resources and Electric Power

Jianquan Yao

North China University of Water Resources and Electric Power

\section{Research Article}

Keywords: Surface plasmon resonance, Wavelength sensitivity, Microchannel, Dual-core

Posted Date: January 3rd, 2022

DOI: https://doi.org/10.21203/rs.3.rs-1173127/v1

License: (c) (i) This work is licensed under a Creative Commons Attribution 4.0 International License. Read Full License

Version of Record: A version of this preprint was published at Plasmonics on April 5th, 2022. See the published version at https://doi.org/10.1007/s11468-022-01637-4. 


\section{A Plasmonic Sensor Based on D-Shaped Dual-Core}

\section{Microchannel Photonic Crystal Fiber}

Pibin Bing ${ }^{\mathrm{a},{ }^{*}}$, Qing Liu ${ }^{\mathrm{b}}$, Guifang $\mathrm{Wu}^{\mathrm{a}}$, Sheng Yuan ${ }^{\mathrm{b}}$, Zhongyang $\mathrm{Li}^{\mathrm{a}}$, Jianquan $\mathrm{Yao}^{\mathrm{a}, \mathrm{c}}$

a College of Electric Power, North China University of Water Resources and Electric Power, Zhengzhou 450045, China

b College of Physics and Electronics, North China University of Water Resources and Electric Power, Zhengzhou 450045, China

c College of Precision Instrument and Opto-Electronics Engineering, Institute of Laser and Opto-Electronics, Tianjin University, Tianjin, 300072, China

\section{Abstract}

In this paper, a dual-core microchannel-based fiber sensor is studied by using finite element method in the visible and near-infrared bands. Plasmonic material gold ( $\mathrm{Au})$ is deposited in microchannel to generate the surface plasmon resonance (SPR) effect, so that sensor can detect the change in RI of its surrounding analyte. Simulation results show that the maximum wavelength sensitivity and resolution are $33600 \mathrm{~nm} / \mathrm{RIU}$ and $2.97 \times 10^{-6} \mathrm{RIU}$ for y polarization in the RI range of 1.33 to 1.44 , respectively. The highest figure of merit (FOM) of the sensor is 961 for y polarization. In addition, we study the effects brought by the structural changes of the fiber sensor, and the results show that the design of "microchannel coating" dramatically improves the refractive index detection ability of the sensor. The D-shaped dual-core microchannel-based photonic crystal fiber sensor proposed in this paper has a simple structure, low manufacturing complexity, and high sensitivity. Combined with external sensing technology, this sensor has great application potential in the fields of biotechnology, medical diagnosis, and environmental protection.

Keywords: Surface plasmon resonance, Wavelength sensitivity, Microchannel, Dual-core

\section{Introduction}

Photonic crystal fiber based on surface plasmon resonance (SPR) is widely applied in biotechnology, medical diagnosis, and environmental protection due to its high sensitivity to variation of RI [1]. SPR is an optical phenomenon, which because evanescent wave will be generated when the electromagnetic wave has a total internal reflection on the surface of the dielectric and evanescent wave can stimulate the surface electron oscillation of metal, and then surface plasmons (SPs) are generated. When the frequency and wavenumber of the SPs and evanescent wave are equal, the two will resonate, which is manifested as a part of the electromagnetic wave is absorbed; The continuous resonating leads to the generation of surface plasmon wave (SPW), which are very sensitive to variation of RI [2]. The variation of RI of the analyte adjacent to the metal film

\footnotetext{
* Corresponding authors. E-mail address: bing463233@163.com (P. Bing).
} 
will affect the propagation constant of the related modes, the resonance peak of the core mode will shift.

Conventional prism-based SPR coupling configurations were Otto and Kretschmann [3,4]. These prism-based SPR coupling configurations, which are composed of various mechanical and optical elements, have disadvantages of bulkiness, unreliability of mechanical structure, high cost, unsuitability for distributed sensing and mass production [5]. The PCF-based SPR sensor has the advantages of miniaturization, flexible design, lightweight, easy integration and real-time monitoring, and is suitable for medical diagnostics and environmental monitoring [6]. Compared with traditional optical fiber, PCF has many superiorities, such as multi-dimensional structure, large mode area, wide coordination range and multi-parameter measurement [7].

The sensor's performance depends mainly on the plasmonic materials and gold, copper, silver, aluminum, etc., are ubiquitous plasmonic materials [8,9]. Silver offers a high resonance peak [10], but suffers from an oxidation issue in an aqueous environment, which means that gold without oxidation issue has better chemical stability, is more suitable for microfluidic analytes than silver. The defect of oxidation can be solved by employing a layer of graphene or indium tin oxide(ITO) or titanium dioxide in nanoscale. This increases not only the fabrication complexity but also manufacturing cost [11].

Recent researches of SPR sensors based on PCF generally comprise two types: internal sensing approach and external sensing approach [12]. The former requires filling the analyte into a specific air hole in the fiber. However, it is not feasible for the real-time sensing application due to the difficulty of filling or emptying the analyte. These disadvantages can be overcome by external sensing technique. Currently, this technique has become popular due to its simple detection [13]. For example, in 2019, H.Thenmozhi et al. proposed an ITO-based D-shaped PCF-SPR sensor showing maximum wavelength sensitivity and resolution of 50000nm/RIU and $4 \times 10^{-4} \mathrm{RIU}$ [14]. In 2019, Md. Nazmus Sakib et al. described a dual-core D-shaped PCF sensor that uses gold as plasmonic material and shows maximum wavelength sensitivity and resolution of $8000 \mathrm{~nm} / \mathrm{RIU}$ and $1.25 \times 10^{-5} \mathrm{RIU}$ [15]. In 2019, Shivam Singh et al. developed a D-shaped PCF sensor with gold and graphene layers. The maximum sensitivity obtained is $33500 \mathrm{~nm} / \mathrm{RIU}$ in the sensing RI range of 1.32 to 1.40 [16]. In 2020, Q.M. Kamrunnahar et al. proposed a simple circular lattice dual-core PCF-based plasmonic sensor [17]. This sensor proffers the maximum wavelength sensitivity and resolution of $11200 \mathrm{~nm} / \mathrm{RIU}$ and $8.92 \times 10^{-6} \mathrm{RIU}$ for wavelength sensing within the RI range of 1.33 to 1.44. In 2020, Hasan Sarker et al. studied a "slotted PCF based plasmonic biosensor", maximum wavelength sensitivity and wavelength resolution are $22000 \mathrm{~nm} / \mathrm{RIU}$ and $4.54 \times$ $10^{-6} \mathrm{RIU}$ [18]. In 2020, Md. Biplob Hossain et al. proposed a D-shaped PCF sensor based on SPR [19]. The proposed sensor shows the highest sensitivity of 
$15000 \mathrm{~nm} / \mathrm{RIU}$ and the maximum resolution is $6.67 \times 10^{-6} \mathrm{RIU}$ in the RI detection range of 1.42 to 1.46 . In 2021, Shivam Singh et al. proposed a dual side-polished PCF RI sensor [1]. Gold, $\mathrm{TiO}_{2}, \mathrm{Si}_{3} \mathrm{~N}_{4}$ are coated on a polished plane fiber surface. The maximum wavelength sensitivity and RI resolution reach $35000 \mathrm{~nm} / \mathrm{RIU}$ and $4.347 \times$ $10^{-6} \mathrm{RIU}$, respectively. The above sensors have obtained high wavelength sensitivity but still exist some problems such as narrow detection range, high preparation complexity, and high cost.

In this paper, the SPR-based dual-core sensor with microchannel has been proposed and numerically analyzed by the finite element method (FEM). The plasmonic metal gold is deposited on the surface of the microchannel to enhance the sensor's performance. Numerical results show maximum wavelength sensitivity and resolution of $33600 \mathrm{~nm} / \mathrm{RIU}$ and $2.97 \times 10^{-6} \mathrm{RIU}$, respectively. Within the RI range of 1.33 to 1.44 , obtained FOM of the sensor is 961RIU ${ }^{-1}$. The proposed sensor not only has high wavelength sensitivity but also low preparation complexity. In addition, due to the advantages of external sensing, the process of filling, discharging, and cleaning the analyte is removed, which greatly enhances the detection efficiency.

\section{Structure design and}

\section{modeling}

In Fig. 1, the cross-section of the proposed PCF sensor can be seen. The designed architecture of the PCF is consisted of air holes arranged in rhombus lattice, and there are six air holes around the optical fiber core. The distances of each air hole to its adjacent air hole in the $\mathrm{y}$ direction and $\mathrm{x}$ direction are $\Lambda$ and $2 \Lambda$, respectively. The diameter of small air holes and big air holes is denoted by $d_{1}$ and $d_{2}$, respectively. The optimized parameters are $\Lambda=2 \mu \mathrm{m}, d_{l}=$ $0.95 \mu \mathrm{m}, \quad d_{2}=1.65 \mu \mathrm{m}$. The three microchannels are above the air holes on both sides of the fiber core, which can boost up the coupling among core and SPP mode, to better realize surface plasmon resonance. Gold is used as a plasmonic material to coat the microchannel, and the thickness of the gold film $\left(t_{g}\right)$ is $40 \mathrm{~nm}$. The vertical height $(h)$ of the microchannel center and the fiber core is $3 \mu \mathrm{m}$. To absorb the radiated energy, a perfectly matched layer (PML) of $2 \mu \mathrm{m}$ thickness has been set around the fiber.

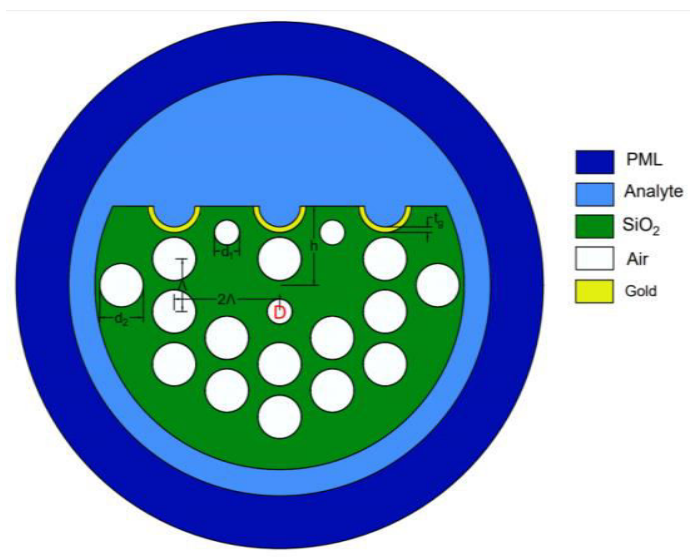

Fig. 1. Schematic of the proposed sensor.

The proposed sensor uses fused silica as background material, and the refractive index of fused silica is given by Sellmeier equation [20]:

$$
n_{S i i_{2}}^{2}(\lambda)=1+\frac{A_{1} \lambda^{2}}{\lambda^{2}-B_{1}}+\frac{A_{2} \lambda^{2}}{\lambda^{2}-B_{2}}+\frac{A_{3} \lambda^{2}}{\lambda^{2}-B_{3}}
$$

where $\lambda$ is the wavelength of the incident light, $\quad \mathrm{A}_{1}=0.696166300, \quad \mathrm{~A}_{2}=$ 
$0.407942600, \mathrm{~A}_{3}=0.897479400, \mathrm{~B}_{1}=$ $4.67914826 \times 10^{-3} \mu \mathrm{m}^{2}, \quad \mathrm{~B}_{2}=$ $1.35120631 \times 10^{-2} \mu \mathrm{m}^{2}, \quad \mathrm{~B}_{3}=$ $97.9340025 \mu \mathrm{m}^{2}$. The dielectric constant of gold is given by Drude model [21]:

$$
\varepsilon_{\mathrm{Au}}(\omega)=\varepsilon^{\infty}-\frac{\omega_{p}^{2}}{\omega\left(\omega+\mathrm{i} \omega_{\tau}\right)}
$$

where $\varepsilon^{\infty}=9.75, \omega_{\mathrm{p}}=1.36 \times 10^{16} \mathrm{rad} / \mathrm{s}, \omega_{\tau}$ $=1.45 \times 10^{14} \mathrm{rad} / \mathrm{s}$.

\section{Results}

\section{Analysis}

and

\section{Discussion}

The parameters of fiber sensor mainly include confinement loss, wavelength sensitivity, resolution, and so on. The sensor proposed in this paper will be studied in the above parameters. The confinement loss is determined by the structure of photonic crystal fiber, such as the number, size, and arrangement of air holes, which also depends on the wavelength of the incident electromagnetic wave. Confinement loss is defined by following equation $[22,23]$ :

$$
\alpha_{\text {loss }}(d B / m)=40 \pi \frac{\operatorname{Im}\left[n_{e f f}\right]}{\ln (10) \lambda}
$$

where $\lambda$ is wavelength in meter, $\operatorname{Im}\left[n_{\text {eff }}\right]$ is the imaginary part of the effective mode RI. Figure 2 displays variation in the loss when the RI of analyte changes from 1.33 to 1.44 . The maximum loss is obtained at resonant wavelengths of $562 \mathrm{~nm}, 572 \mathrm{~nm}, 584 \mathrm{~nm}, 598 \mathrm{~nm}, 614 \mathrm{~nm}$, $633 \mathrm{~nm}, 655 \mathrm{~nm}, 681 \mathrm{~nm}, 721 \mathrm{~nm}, 760 \mathrm{~nm}$, $1077 \mathrm{~nm}, 1413 \mathrm{~nm}$. As we can see in Fig. 2 , when the RI of analyte changes from 1.33 to 1.44 , confinement loss increases from $288 \mathrm{~dB} / \mathrm{m}$ to $3307 \mathrm{~dB} / \mathrm{m}$ with red-shift of resonance peak. The analytes with higher RIs have greater capability to influence the SPP mode effective refractive index. The phase-matching point of the core mode and SPP mode is red-shifted with the increase of refractive index; Under the condition of constant refractive index, the effects of different wavelengths on the gold film are different. As the RI of the analyte increases, it is closer to the RI of the fiber core, which will cause the strong coupling of the core mode and SPP mode, the confinement loss increases correspondingly.

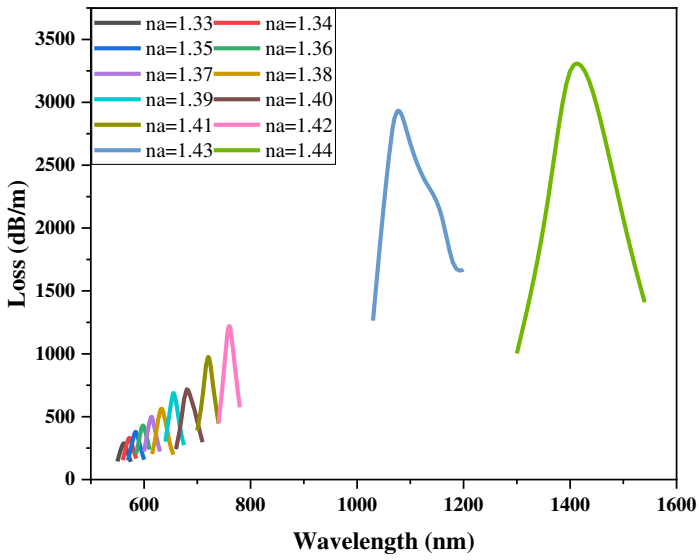

Fig. 2. Loss curves of proposed PCF sensor for analyte RI from 1.33 to 1.44 .

Figure 3(a) depicts the dispersion relationship for the loss curve, y-polarized core mode and SPP mode for an analyte RI 1.42. We mainly analyze y polarization because of the stronger interaction between $y$ polarization and gold film. The red line represents the variation curve of the confinement loss for y-polarized core mode, while the purple and green line show the effective RI of SPP mode and the real part of the effective RI of core mode, respectively. In Fig. 3(a), purple line and green line intersect at resonant wavelength position $760 \mathrm{~nm}$ for the analyte RI of 1.42 , which means that the 
phase matching condition is fulfilled, the coupling between the SPP mode and core mode is achieved, and maximum energy is transferred from the core mode to the SPP mode. The effective RI of SPP mode decreases faster than that of core mode, the coupling intensity of the two modes decreases, and the loss decreases gradually when the two modes are far from the intersection. Figure 3(b-d) displays field distribution of SPP mode, y-polarized and $\mathrm{x}$-polarized core-guided mode for the analyte RI of 1.42 .

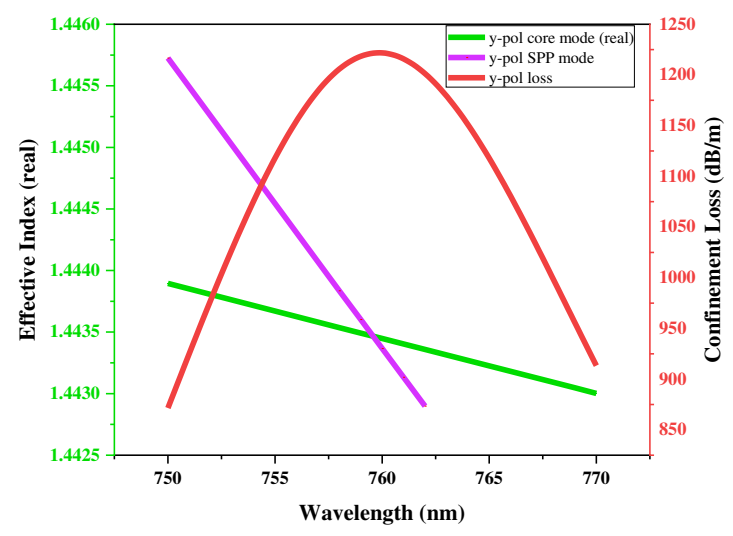

(a)

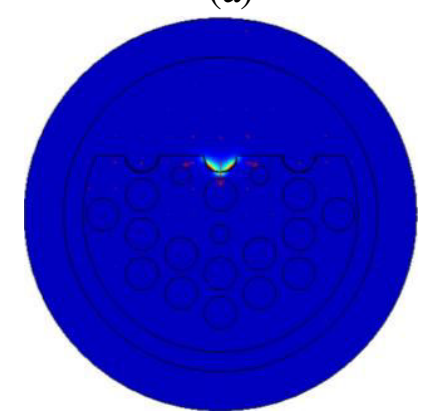

(b)

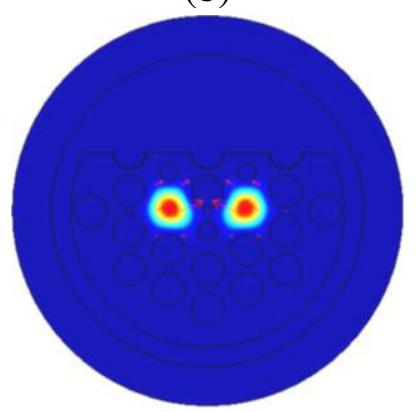

(c)

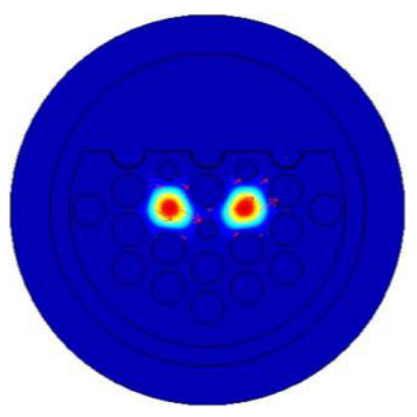

(d)

Fig. 3. (a)Dispersion relationship of y-polarized core mode, SPP mode and loss curves. (b)SPP mode, (c)y-polarized core mode, (d) x-polarized core mode for analyte RI 1.42 .

Wavelength sensitivity (WS) is an important parameter to describe the performance of PCF sensor, which indicates the shift of the sensor to the resonance peaks of different RI analytes. Wavelength sensitivity is defined by using wavelength interrogation method [24]:

$$
S_{\lambda}(n m / R I U)=\frac{\Delta \lambda_{\text {peak }}}{\Delta n_{a}}
$$

Figure 4 depicts the variation curves of wavelength sensitivity and resonance wavelength. The black line in Fig. 4 shows that the resonance wavelength diminishes with the increase of RI. However, this paper argues that the resonance wavelength will not increase infinitely, because there are often multiple peak values in the loss curve. The first peak in the visible or near-infrared bands is usually selected as the resonance peak. With the increase of wavelength, there may be secondary peak near the main resonance peak, and the secondary peak value is also close to the peak value of the main peak, which will affect our judgment of the main peak and resonance wavelength. In addition, there may be cases where the 
resonance peak fades out and the resonance wavelength does not exist. The shift of resonance wavelength is $10 \mathrm{~nm}, 12 \mathrm{~nm}, 14 \mathrm{~nm}, 16 \mathrm{~nm}, 19 \mathrm{~nm}, 22 \mathrm{~nm}$, $26 \mathrm{~nm}, 40 \mathrm{~nm}, 39 \mathrm{~nm}, 317 \mathrm{~nm}, 336 \mathrm{~nm}$ at analyte RI of 1.34 to 1.44 ; The red line shows the variation of WS, and WSs obtained at RIs of 1.42 and 1.43 is $31700 \mathrm{~nm} / \mathrm{RIU}$ and $33600 \mathrm{~nm} / \mathrm{RIU}$, respectively.

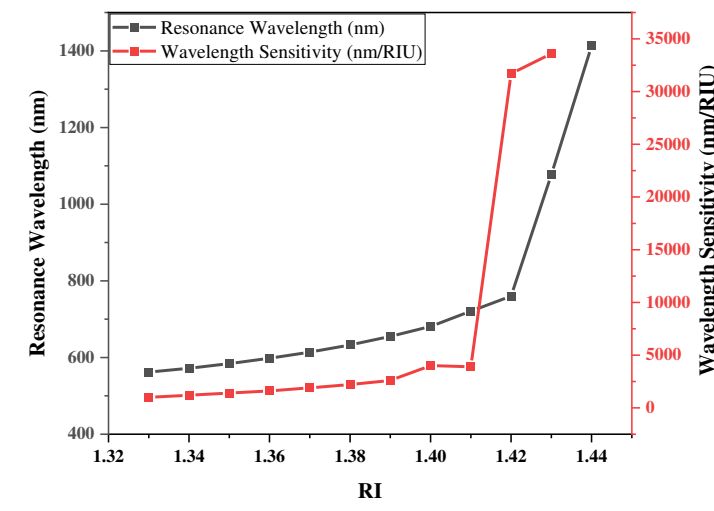

Fig. 4. Variation curves of resonance wavelength and WS for analyte RI from 1.33 to 1.44 .

Sensor resolution is a parameter that is used to measure the lowest change of analyte RI that can be detected by the sensor. The sensor resolution can be defined as [25]:

$$
R(R I U)=\frac{\Delta n_{a} \times \Delta \lambda_{\text {min }}}{\Delta \lambda_{\text {peak }}}
$$

Where $\Delta n_{a}$ is the variation of the analyte RI, $\Delta \lambda_{\min }$ is the minimum spectral resolution, and $\Delta \lambda_{\text {peak }}$ is the shift of resonance wavelength. The maximum resolution of proposed sensor is $2.97 \times 10^{-6} \mathrm{RIU}$ with $\Delta n_{a}=0.01 \mathrm{~nm}$, $\Delta \lambda_{\text {min }}=0.1 \mathrm{~nm}$ and $\Delta \lambda_{\text {min }}=336 \mathrm{~nm}$. The value suggests the sensor can detect the analyte RI with a change in the order of $10^{-6}$ for analyte RI of 1.43 .

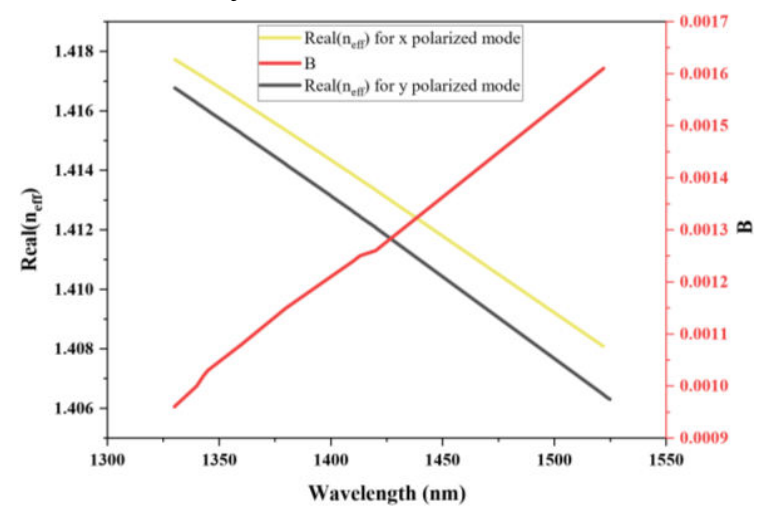

Fig. 5. Variation curve for birefringence of the analyte RI 1.44. The variation curves of $\operatorname{real}\left(\mathrm{n}_{\mathrm{eff}}\right)$ of the $\mathrm{x}$ - and $\mathrm{y}$-polarized mode with wavelength for analyte RI 1.44.

Preserving the polarization state is important in sensing and long-distance communication. The ease of birefringence is due to the flexible structural design and optical properties of PCF. There are two types of core modes: $\mathrm{x}$ - and $\mathrm{y}$-polarized core modes. The real part of the effective RI of the $\mathrm{X}$-polarized core mode is different from that of the $y$-polarized core mode. Birefringence (B) is defined as the difference in the RI real parts of the core modes for the two orthogonal polarizations [26]:

$$
B=\left|\operatorname{Re}\left(n_{e f f}^{x}\right)-\operatorname{Re}\left(n_{e f f}^{y}\right)\right|
$$

Figure 5 reveals variation curves of birefringence, the RI real parts of $\mathrm{x}$ and $y$ polarizations for analyte RI of 1.44. The birefringence increases gradually and the real part of the effective RI of both polarizations decreases gradually

Table 1. FOM for different analyte RI

\begin{tabular}{|c|c|c|c|c|c|c|c|c|c|c|c|c|}
\hline RI & 1.33 & 1.34 & 1.35 & 1.36 & 1.37 & 1.38 & 1.39 & 1.40 & 1.41 & 1.42 & 1.43 & 1.44 \\
\hline FOM & 40 & 50 & 54 & 64 & 68 & 73 & 87 & 98 & 111 & 961 & 210 & N/A \\
\hline
\end{tabular}
with the increase of wavelength. Birefringence of $1.6 \times 10^{-3}$ can be


obtained at $1523 \mathrm{~nm}$ wavelength for analyte RI of 1.44 .

In addition, the figure of merit (FOM) can be calculated as the ratio of WS to full width at half maximum (FWHM) to evaluate the overall performance of a sensor [27-29]. When the WS increases, the FWHM should be as small as possible, so that when the RI of analyte changes, we can observe the red shift or blue shift of the loss peak clearly. Table 1 shows the obtained FOM for different analyte RI.

\section{Analysis and Discussion of}

\section{Structure Parameters}

The changes in the structure of the sensor will affect the parameters (confinement loss and resonance wavelength) of the sensor. This chapter will discuss the influence brought by the change of structural parameters, including the size of the air hole, the position of the gold coating, and the vertical distance $h$ between the polished plane and the fiber core.

Figure 6 shows the influence on the loss when the diameter of the air hole D changes from $0.95 \mu \mathrm{m}$ to $2.1 \mu \mathrm{m}$. It can be observed that the loss peak value gets higher with increasing diameter value. When the diameter of air hole D is large, the energy of the two cores has little influence on each other, and the energy in the core produce a stronger SPR effect with the gold film, which results in a larger loss peak. Compared with the case of larger air hole D diameter, the case of smaller D diameter leads to larger dual-core, which means that the dual-core has a stronger ability to limit energy. In this case, the smaller the SPR effect, the smaller the loss. When the diameter of the air hole D is constant, the phase-matching point of the core mode and SPP mode is red-shifted with the increase of refractive index, which results in the resonance peak red-shifted. As the RI of the analyte increases, it is closer to the RI of the fiber core, which will cause the strong coupling of the core mode and SPP mode, the loss value increases correspondingly.

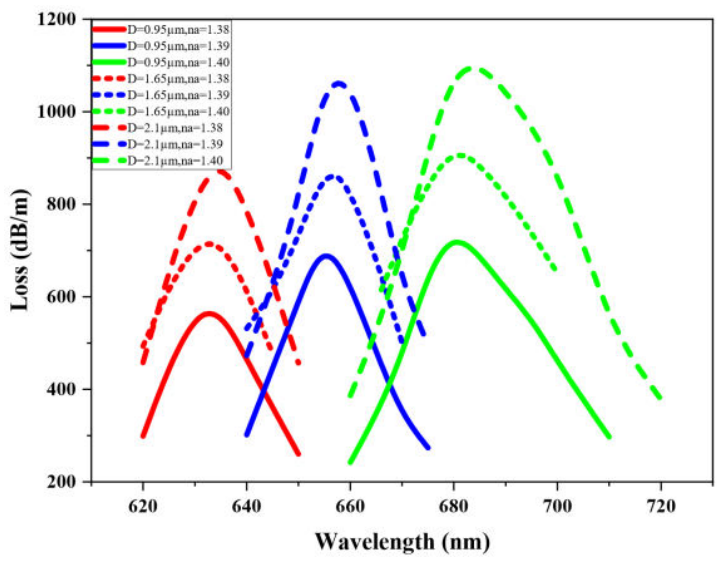

Fig. 6. Loss peak variation with air hole diameter for the analyte RI from 1.38 to 1.40 .

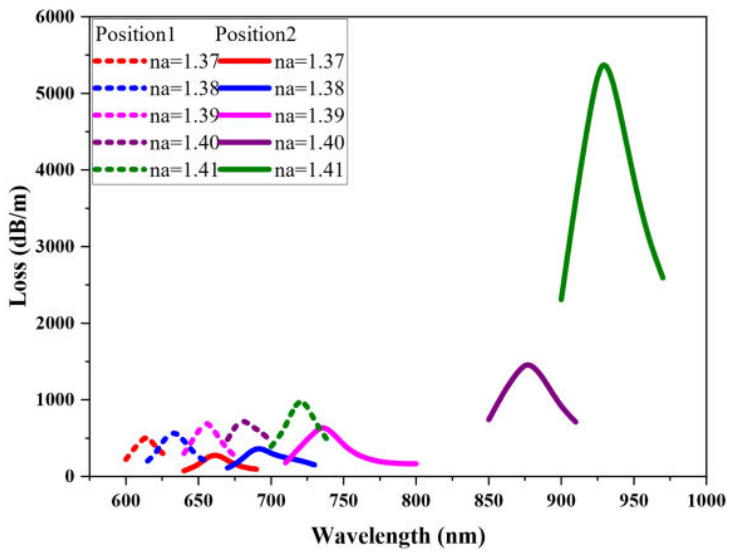

(a) 


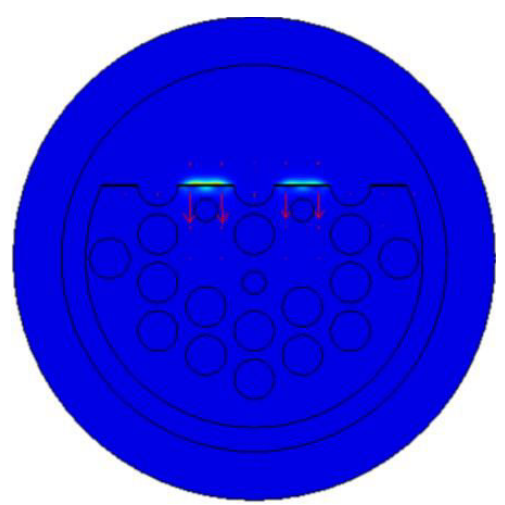

(b)

Fig. 7. (a)Variation in loss peaks for sensors with microchannel coating and polished plane coating. (b) SPP mode of the sensor with polished plane coating.

The coating position of the gold film also affects the performance of sensor directly. Figure 7(a) reveals the loss curves of sensors with different coating positions (inner wall of microchannel, polished plane). Figure 7(b) depicts SPP mode for the sensor coated in the microchannel. Position1 and Position2 are used to represent the microchannel coating and polished plane coating, respectively. It can be seen from the Fig. 7(a) that when the analyte RI changes from 1.38 to 1.41 , the loss curves of the two coating methods both show that the resonance peak red-shifts with the increase of wavelength. For the same analyte RI, the difference between the two coating methods is that the loss of polished plane coating is smaller than that of microchannel coating, which is due to the polished plane coating method increases the distance between the gold film and the fiber core and weakens the resonance intensity between the incident electromagnetic wave and metallic electrons. With an increment of analyte RI, the loss peak value of polished plane coating increases faster than that of microchannel coating, which is related to broader passage along the y direction. Figure 8 displays the loss curves of the sensor with microchannel coating (Position1), both microchannel and polished plane coating (Position3). Combining with Fig. 7, it can be inferred that microchannel coating and polished plane coating exhibit a larger resonance shift than that of both microchannel and polished plane coating, indicating that the two coating methods of microchannel coating and polished plane coating improve the sensor's performance. In addition, our data show that the microchannel coating method has better detection ability than the polished plane coating method when the analyte RI > 1.41. Considering the characteristics of the loss curve of the sensor with polished plane coating, it is suitable for the sensing range of low RI, and its detection potential can be developed by optimizing the structural parameters.

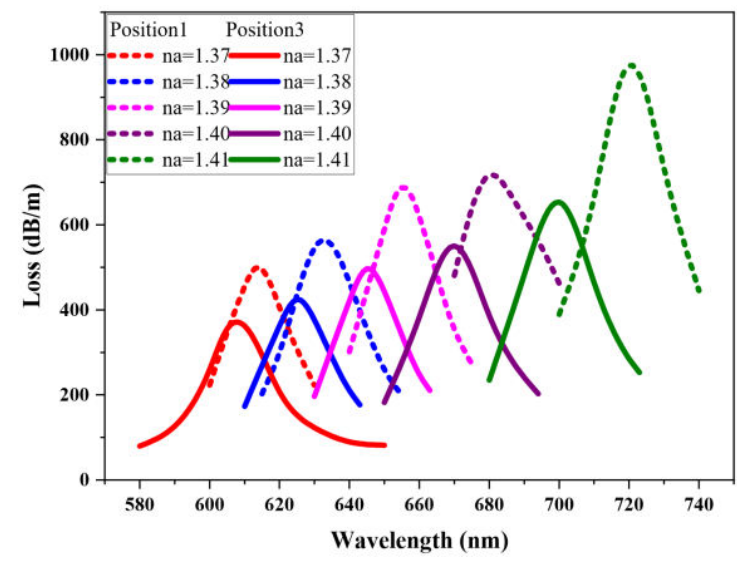

Fig. 8. Variation in loss peaks for sensors with microchannel coating, both microchannel and polished plane coating. 


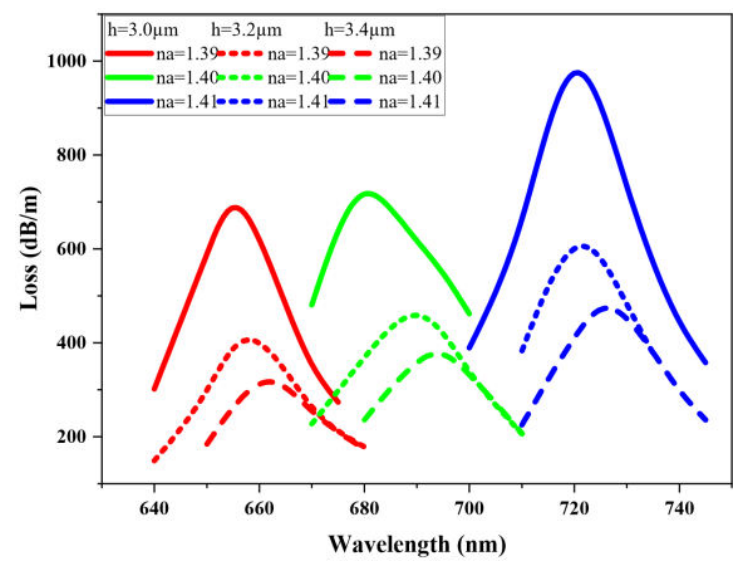

Fig. 9. Loss peak variation for the changes of distance $h$.

From Fig. 9 we can see that the vertical distance $h$ between the polished plane and the fiber center has significant impact on the sensor property. It can be observed that when the distance $h$ increases from $3.0 \mu \mathrm{m}$ to $3.4 \mu \mathrm{m}$, the loss peak value decreases. This indicates that the further distance between the center and the gold film, the less energy transferred from the core to the gold film, which means that the coupling intensity between the SPP mode and the core-guided mode gradually decreases, and the loss decreases accordingly. In addition, the changed phase matching point of core-guided mode and SPP mode with the increase of the analyte RI resulting in a red-shifted resonance peak. The stronger coupling degree of the core-guided mode and the SPP mode is related to the fact that the RI of the analyte is closer to the effective RI of the fiber core, and the loss peak value increases gradually.

\section{Conclusion}

An SPR-based microchannel dual-core PCF sensor is proposed and analyzed numerically with an analyte detection range of 1.33 to 1.44 .
Simulation and analysis depict that the maximum wavelength sensitivity, resolution was obtained as $33600 \mathrm{~nm} / \mathrm{RIU}$ and $2.97 \times 10^{-6} \mathrm{RIU}$, respectively, for the sensing range of 1.33 to 1.44 . Furthermore, the sensor can achieve a birefringence of $1.6 \times 10^{-3}$ and a maximum FOM of $961 \mathrm{RIU}^{-1}$ for the y polarization. Moreover, not only the performance of the sensor was improved, but also the convenient detection method is realized because of the microchannel coating and dual-core structure. The sensor can be directly immersed in the microfluidic analyte for detection, which solves the problem of analyte filling and cleaning. The sensor proposed in this paper has good performance which has potential applications in biotechnology and chemical sensing fields.

\section{Declaration}

Competing Interest The authors declare no competing interests.

Funding This research was funded by the National Natural Science Foundation of China (Grant No. 61601183, 31671580), the Key Technologies Research and Development Program of Henan Province (Grant No. 202102210390), and Young Backbone Teachers in University of Henan Province (Grant No. 2020GGJS099).

Availability of data Data may be obtained from the authors upon reasonable request.

Ethics approval Approved.

Consent to participate Approved.

Consent for publication Approved.

Code availability Available.

Authors' contributions Pibin Bing 
concieved the concept. Qing Liu fabricated the model and conducted the simulation. Guifang $\mathrm{Wu}$ contributed to the data analysis. Pibin Bing, Qing Liu wrote the manuscript. Sheng Yuan, Zhongyang $\mathrm{Li}$ and Jianquan Yao discussed the results and contributed to the writing of the manuscript. Pibin Bing supervised the project..

\section{References}

1. Singh S, Prajapati YK (2021) Highly sensitive dual-core symmetrical side-polished modified D-shaped SPR based PCF refractive index sensor with deeply etched micro openings. Optik 235:166657

2. Sakib MN, Hossain MB, Al-tabatabaie $\mathrm{KF}$ et al (2019) High performance dual core D-shape PCF-SPR sensor modeling employing gold coat. Results in Physics 15:102788

3. Otto A (1968) Excitation of nonradiative surface plasma waves in silver by the method of frustrated total reflection. Zeitschrift für Physik A Hadrons and nuclei 216:398-410.

4. E. Kretschmann, R. Heinz (1968) Radiative decay of non radiative surface plasmons excited by light. Zeitschrift für Naturforschung A 23:2135-2136.

5. Paul AK, Mollah MA, Hassan MZ et al (2021) Graphene-Coated Highly Sensitive Photonic Crystal Fiber Surface Plasmon Resonance Sensor for Aqueous Solution: Design and Numerical Analysis. Photonics 8(5):155

6. Rahman MM, Molla MA, Paul AK et al (2020) Numerical investigation of a highly sensitive plasmonic refractive index sensor utilizing hexagonal lattice of photonic crystal fiber. Results Phys
18:103313

7. Al Mahfuz M, Hossain MA, Haque E et al (2020) Dual-Core Photonic Crystal Fiber-Based Plasmonic RI Sensor in the Visible to Near-IR Operating Band. Ieee Sens J 20(14):7692-7700

8. Liu YD, Chen HL, Guo Y et al (2020) Ultra-high sensitivity plasmonic sensor based on D-shaped photonic crystal fiber with offset-core. Optik 221:165309

9. Khaleque A, Mironov EG, Osorio JH et al (2017) Integration of bow-tie plasmonic nano-antennas on tapered fibers. Opt Express 25(8):8986-8996

10. Sunny SMA, Ahmed T, Hiam SM et al (2021) Highly sensitive externally metal coated plasmonic refractive index sensor based on photonic crystal fiber. Optik 243:167482

11. Wang Q, Wang XZ, Song $\mathrm{H}$ et al (2020) A dual channel self-compensation optical fiber biosensor based on coupling of surface plasmon polariton. Opt Laser Technol 124:106002

12. Ahmed T, Paul AK, Anower MS et al (2019) Surface plasmon resonance biosensor based on hexagonal lattice dual-core photonic crystal fiber. Appl Optics 58(31):8416-8422

13. Haque E, Mahmuda S, Hossein MA et al (2019) Highly Sensitive Dual-Core PCF Based Plasmonic Refractive Index Sensor for Low Refractive Index Detection. Ieee Photonics J 11(5):1-9

14. Thenmozhi H, Rajan MSM and Ahmed K (2019) D-shaped PCF sensor based on SPR for the detection of carcinogenic agents in food and cosmetics. Optik 180:264-270

15. Sakib MN, Hossain MB, Al-tabatabaie KF et al (2019) High performance dual core D-shape PCF-SPR sensor modeling 
employing gold coat. Results Phys 15:102788

16. Singh S and Prajapati YK (2019) Highly sensitive refractive index sensor based on D-shaped PCF with gold-graphene layers on the polished surface. Appl Phys a-Mater 125(6):1-7

17. Kamrunnahar QM, Mou JR and Momtaj M (2020) Dual-core gold coated photonic crystal fiber plasmonic sensor: Design and analysis. Results Phys 18:103319

18. Sarker H, Faisal $M$ and Mollah MA (2021) Slotted photonic crystal fiber-based plasmonic biosensor. Appl Optics 60(2):358-366

19. Hossain MB, Hossain MS, Islam SMR et al (2020) Numerical development of high performance quasi D-shape PCF-SPR biosensor: An external sensing approach employing gold. Results Phys 18:103281

20. Bing PB, Sui JL, Wu GF et al (2020) Analysis of Dual-Channel Simultaneous Detection of Photonic Crystal Fiber Sensors. Plasmonics 15(4):1071-1076

21. Kurihara K, Nakamura K, Hirayama E et al (2002) An absorption-based surface plasmon resonance sensor applied to sodium ion sensing based on an ion-selective optode membrane. Analytical chemistry 74:6323-6333

22. Wu QL, Zhao Y, Zhang YN et al (2021) Characteristics of a new multi-channel sensing device based on C-type photonic crystal fibers. Opt Laser Technol 134:106622

23. Yan X, Fu R, Cheng TL et al (2021) A Highly Sensitive Refractive Index Sensor Based on a V-Shaped Photonic Crystal Fiber with a High Refractive Index Range. Sensors-Basel 21(11):3782

24. Chen X, Xia L and Li C (2018) Surface Plasmon Resonance Sensor Based on a
Novel D-Shaped Photonic Crystal Fiber for Low Refractive Index Detection. Ieee Photonics J 10(1):1-9

25. Islam MS, Sultana J, Rifat AA et al (2018) Dual-polarized highly sensitive plasmonic sensor in the visible to near-IR spectrum. Opt Express 26(23):30347-30361

26. Liu C, Su W, Wang F et al (2018) Birefringent PCF-Based SPR Sensor for a Broad Range of Low Refractive Index Detection. IEEE Photonics Technology Letters 30(16):1471-1474

27. Liu C, Su W, Liu Q et al (2018) Symmetrical dual D-shape photonic crystal fibers for surface plasmon resonance sensing. Opt Express 26(7):9039-9049

28. Peng Y, Hou J, Zhang Y et al (2013) Temperature sensing using the bandgap-like effect in a selectively liquid-filled photonic crystal fiber. Opt Lett 38(3):263-265

29. Srivastava T, Das R and Jha R (2013) Highly Sensitive Plasmonic Temperature Sensor Based on Photonic Crystal Surface Plasmon Waveguide. Plasmonics $8(2): 515-521$ 